\title{
Introduction to Social Netorking and Communities Minitrack
}

\author{
Karine Nahon \\ Interdisciplinary Centre Herzliya and \\ University of Washington \\ karineb@uw.edu
}

\author{
Dan Suthers \\ University of Hawaii at Manoa \\ suthers@hawaii.edu
}

We call for papers that investigate social networks and communities supported and/or complemented by social media and other social technologies for work, learning, socializing, economic or political processes, and/or that address theory, design, practices, use or evaluation of the use of social technologies. 'Social networks and communities' refers broadly to social phenomena that may range from fully virtual to geographically based communities, and includes communities of interest, communities of practice, epistemic communities and networked advocacy, as well as other phenomena emerging within networks of individuals such as networked spread of influence ("virality").

We solicit papers that examine relationships between social phenomena and technologies, including how social phenomena are embedded or emerge within technological settings, how communities use technologies to further their goals, or how technologies otherwise influence or are appropriated by social phenomena and entities. Individual actors as the unit of analysis may also be included as long as communities and technologies are involved; e.g., how individuals relate to communities via technologies.
In order to leverage the interdisciplinary nature of HICSS and enable dialogue between social and technological disciplines, papers may be motivated by a broad range of theory-driven or data-driven perspectives and methods, including for example but not limited to social network analysis, content analysis, ethnographic investigations, and agent-based simulations. We particularly encourage papers that:

- advance our understanding of social network growth, formation, structure and outcomes through social media;

- explore how socio-technical affordances relate to social media use and outcomes;

- examine how information and misinformation spread in social media and networks, and how algorithms, bots, and social media design affect this spread;

- evaluate design of social media technologies and practices for effective community development and maintenance; and

- develop theories, models and principles of social media design, use and outcomes. 